\title{
Predictive value of HEART score in the outcome of acute coronary syndrome and disposition
}

\author{
Ahmad Abbasian $^{1^{\circledR}}$, Maryam Beladi ${ }^{\circledR}$, Elnaz Vahidi ${ }^{*}{ }^{\circledR}$, Amirhosein Jahanshir $^{(\mathbb{D}}$, Javad Seyedhosseini $^{\circledR}$ \\ 1 Emergency Department, Imam Khomeini Hospital Complex, Tehran University of Medical Sciences, Tehran, Iran \\ 2Emergency Department, Shariati Hospital, Tehran University of Medical Sciences, Tehran, Iran
}

\author{
Received: 13 April 2021 \\ Accepted: 16 July 2021 \\ Published online: 23 July 2021 \\ *Corresponding author: \\ Elnaz Vahidi, \\ Emergency Department, Shariati \\ Hospital, Tehran University of \\ Medical Sciences, Tehran, Iran \\ Tel: + 989125948762; \\ Email: evahidi62@yahoo.com, \\ e-vahidi@sina.tums.ac.ir \\ Competing interests: None. \\ Funding information: None. \\ Citation: Abbasian A, Beladi M, \\ Vahidi E, Jahanshir A, Seyedhosseini \\ J. Predictive value of HEART score \\ in the outcome of acute coronary \\ syndrome and disposition. Journal \\ of Emergency Practice and Trauma \\ 2022; 8(1): 19-25. doi: 10.34172/ \\ jept.2021.17.
}

\begin{abstract}
Objective: Disposition in acute coronary syndrome (ACS) is pivotal in an emergency department (ED). HEART score is a recent scoring system for finding primary endpoints in undetermined ACS. This study aimed at evaluating the predictive value of HEART score in ACS outcome and disposition.

Methods: In this prospective study, all patients with chest pain presentation compatible with our inclusion criteria referring to ED were enrolled during one year. Demographic data, triage level, hospital length of stay, admission ward, coronary angiography result, HEART score, thrombolysis in myocardial infarction (TIMI) score, 1-month primary ACS endpoints and major adverse cardiac events (MACE) were evaluated.

Results: In our studied population (200 cases), 49 patients (24.5\%) had at least one score for MACE. Comparing the prognostic values of TIMI vs HEART score in MACE revealed that the HEART had a larger AUC. The best cut-off point of HEART score in MACE prediction was calculated to be $\geq 5$. There was a statistically significant relation between HEART score and hospital length of stay. The higher the HEART score, the more probability of patients being admitted to either hospital cardiac ward or coronary care unit (CCU). There was a significant relationship between the triage level and HEART score. Patients with higher HEART score had more acuity (lower triage level 1 or 2).

Conclusion: HEART predicted MACE better than TIMI in low risk ACS. Patients with higher HEART score were more admitted to the hospital with longer hospital stay and patients with lower HEART score had higher triage level with less acuity.

Keywords: Acute coronary syndrome, HEART score, TIMI score, MACE score, Disposition
\end{abstract}

\section{Introduction}

Chest pain is one of the most common presentations in the emergency department (ED), about $6.3 \%$ of ED visits (1). There is a great deal of differential diagnosis when facing a patient with the chest pain, acute coronary syndrome (ACS), pulmonary emboli, vascular events, and noncardiac presentations (2,3). In an overcrowded environment such as an $\mathrm{ED}$, it is essential and vital to find patients with major cardiac events (especially acute myocardial infarction, AMI) as soon as possible in order to limit the adverse events and mortality with the best management. In the meantime, detecting low risk patients with atypical presentations is another medical worrisome. One must precisely decide with courage to discharge these patients safely to home and be sure that no cardiac threats occur in the future. It is evident that less than $25 \%$ of all chest pain patients have truly ACS (4). By finding low risk ACS, we can reduce health care system expenses, hospital length of stay, occupied hospital bed and disease burden (5-7). In the United States of America, there is an annual rate of more than 7 million ED visits because of suspected ACS, but $3 / 4$ of these cases are finally found to have noncardiac chest pain (8). Evaluation and treatment of these patients cost American health care system more than 10 billion dollars each year (9). On the other hand, it has been reported that almost $2 \%-4 \%$ of AMI patients are being discharged from ED without the correct diagnosis. This is one of the major judicial and legal issues for emergency physicians (10).

Clinicians use different scoring tools besides patient history, electrocardiography (ECG), echocardiography (Echo) and serum biomarkers (notably troponin I [Tr I]). Thrombolysis in myocardial infarction (TIMI) (11), Global Registry of Acute Cardiac Events (GRACE) (12) and HEART score (3) are now widely used to diagnose ACS and predict major adverse cardiac events (MACE) 
(13-15). MACE includes AMI, percutaneous coronary infection (PCI), coronary artery bypass graft (CABG) and death. HEART score can be used in low risk patients whose diagnosis of ACS is not yet confirmed. Clinicians can decide whether to release and discharge patients or admit them for further evaluations (16). In previous studies, it is reported that there is a significant difference in the predictive value of HEART score comparing to other scoring tools $(2,15)$. Literature still needs more research in this field to estimate the exact value of HEART score in patients' disposition in ED.

In this prospective study, we evaluate the diagnostic and prognostic values of HEART score in an ED full of cases with multiple underlying diseases. We also evaluate patients' disposition and triage level according to HEART score.

\section{Methods}

This was a diagnostic accuracy study performed during 1 year in 3 major general hospitals of Tehran University of Medical Sciences (TUMS). The inclusion criterion was age older than 18 years old with acute chest pain of at least 5 minutes duration referring to the ED. The exclusion criteria were patients with: evidence of ST segment elevation in ECG, syncope, dyspnea, unusual complaints and angina equivalents, tachy- or brady-dysrhythmia, traumatic chest pain, missing data, incomplete or inaccurate documentation. The method and study process were explained to patients. Informed written consent was taken from all patients who decided to participate in our study. The study was approved by the ethics committee of TUMS (ID: IR.TUMS.IKHC.REC.1396.3888).

Demographic data, cardiovascular risk factors, TIMI and HEART scores, MACE outcome, hospital length of stay, disposition and triage level based on Emergency Severity Index (ESI) were all evaluated and documented in a predesigned checklist. Patients were initially admitted to ED and finally were either discharged (less than 24 hours after admission), stayed longer in ED, cardiac hospital ward or coronary care unit (CCU). All patients were followed closely for further 30 days after admission via face-to-face appointment or phone call and primary ACS endpoints (MACE) were documented. One-month outcome was assessed by either face-to-face interview in follow-up sessions or phone call. emergency physicians enrolled patients, observed the treatment course and completed the checklist.

Our primary outcome was to compare the prognostic value of HEART score vs TIMI score in MACE prediction. Our secondary outcomes were evaluating the relationship of HEART score with patients' triage level, disposition and hospital length of stay.

Based on reference number (17), we calculated a sample size of 200 patients, by considering $P=0.22, \mathrm{~d}=0.06$, $\alpha=0.05$ and power $=80 \%$. After gathering all data, they were inserted into SPSS (version 25.0) and Stata (version 15.0) software packages. The descriptive indices such as frequency (percentage) and mean (standard deviation, $\mathrm{SD}$ ) were used to express the results. Chi-square test, independent $t$ test, ANOVA analysis of variance and Pearson correlation analysis were used accordingly. The predictive value of scoring systems was evaluated by area under receiver operating characteristic (ROC) curve (AUC) with $95 \%$ confidence interval (CI). The best cut-off point of scoring systems based on the best sensitivity and specificity was determined by $95 \%$ CI (Youden index). The level of significance was 0.05 .

\section{Results}

In this study, we evaluated 237 suspected cases of ACS during 6 months. Twenty cases were excluded and 17 cases were missed to follow-up (Figure 1). Data of 200 remaining cases were complete and we enrolled them in our study. In our sample size, 119 patients were males and 81 patients were females. The age range was from 24 to 88 years with the mean \pm SD of $58.0 \pm 12.5$ years old. The mean time of hospital length stay was $3.6 \pm 2.0$ days ( 1 to 25 days). Most of our patients were admitted shorter than 5 days. Most of our cases were admitted to the ED (45.0\%)

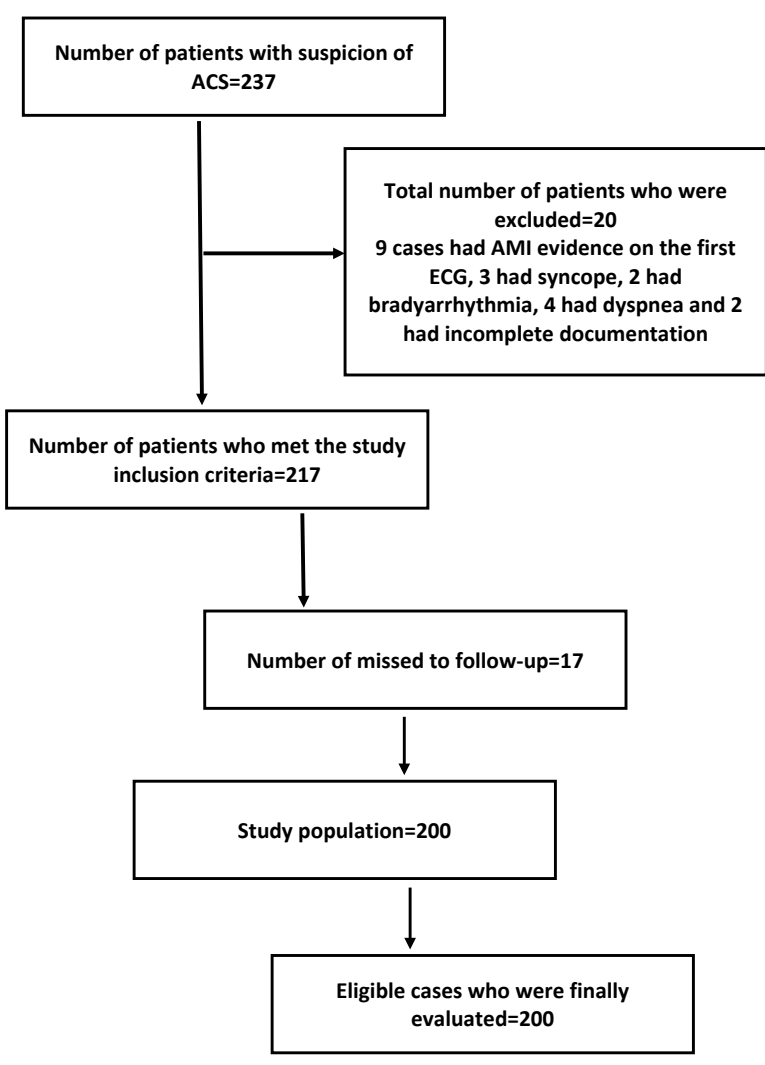

Figure 1. Flow chart of the study. 
with the triage level of $2(91.5 \%)$. Among all suspected ACS cases, 49 patients (24.5\%) had at least 1 positive score for MACE. PCI was the most common MACE (in 34 patients (17.0\%)). Baseline data is shown in Table 1.

We evaluated the relationships of our study variables with MACE score. We observed that there was a significant association between triage level and MACE score. Patients with more ACS acuity (level 1 triage) had more probability of MACE occurrence $(P=0.019)$.

Patients in whom MACE happened had significantly longer hospital length of stay $(P<0.001)$. Incidence of

Table 1. Baseline and demographic data

\begin{tabular}{|c|c|c|}
\hline \multicolumn{3}{|l|}{ Variable } \\
\hline \multirow{2}{*}{ Gender, No. (\%) } & Male & $119(59.5)$ \\
\hline & Female & $81(40.5)$ \\
\hline \multicolumn{2}{|l|}{ Age $(y)$, Mean \pm SD } & $58.0 \pm 12.5$ \\
\hline \multicolumn{2}{|c|}{ Hospital length of stay (day), Mean \pm SD } & $3.6 \pm 2.0$ \\
\hline \multirow{3}{*}{$\begin{array}{l}\text { Triage level, } \\
\text { No. (\%) }\end{array}$} & 1 & $5(2.5)$ \\
\hline & 2 & $183(91.5)$ \\
\hline & 3 & $12(6.0)$ \\
\hline \multirow{4}{*}{$\begin{array}{l}\text { Disposition, } \\
\text { No. }(\%)\end{array}$} & ED longer than 24 hours & $10(5.0 \%)$ \\
\hline & Cardiac ward & $74(37.0)$ \\
\hline & $\mathrm{CCU}$ & $34(17.0)$ \\
\hline & Discharged shorter than 24 hours & $82(41.0)$ \\
\hline \multirow{5}{*}{$\begin{array}{l}\text { MACE outcome, } \\
\text { No. }(\%)\end{array}$} & Negative & $151(75.5)$ \\
\hline & CABG & $12(6.0)$ \\
\hline & AMI & $1(0.5)$ \\
\hline & $\mathrm{PCl}$ & $34(17.0)$ \\
\hline & Cardiac death & $2(1.0)$ \\
\hline
\end{tabular}

Abbreviations: MACE, major adverse cardiac events; AMI, acute myocardial infarction; $\mathrm{PCl}$, percutaneous coronary infection; $\mathrm{CABG}$, coronary artery bypass graft; $\mathrm{CCU}$, coronary care unit; $\mathrm{ED}$, emergency department. abnormal (positive) scores in all HEART score components was significantly more in patients with MACE occurrence $(P<0.05)$. HEART score had a significant relationship with MACE score. Patients with higher HEART score had more probability of MACE occurrence $(P<0.001)$.

Some cases had indeterminate history of coronary stenosis $>50 \%$. Thus, we excluded these patients in TIMI score assessment and we evaluated 176 patients for this score. Incidence of abnormal (positive) scores in all TIMI score components (except age older than 65 years old) was significantly more in patients with MACE occurrence $(P<0.05)$. TIMI score had a significant relationship with MACE score. Patients with higher TIMI score had more probability of MACE occurrence $(P<0.001)$. Data is depicted in Table 2.

The value of HEART score in MACE prediction based on AUC (ROC curve) was estimated to be 0.850 (95\% CI: 0.792 to 0.896 ). HEART score $\geq 4$ was the cut-off point in MACE prediction with the highest sensitivity and HEART score $\geq 7$ was the cut-off point in MACE prediction with the highest specificity. The best cut-off point was calculated to be $\geq 5$.

The value of TIMI score in MACE prediction based on AUC (ROC curve) was estimated to be 0.814 (95\% CI: 0.749 to 0.869 ). TIMI score $\geq 3$ was the cut-off point in MACE prediction with the highest sensitivity and TIMI score $\geq 5$ was the cut-off point in MACE prediction with the highest specificity. The best cut-off point was calculated to be $\geq 3$. Data is shown in Table 3 .

The result of predictive value comparison of HEART vs TIMI scores revealed that AUC for HEART was bigger than TIMI, although this difference was not significant $(P=0.133)$ (Figure 2). For this analysis, as we mentioned before, we could enroll data of 176 patients in TIMI score.

We also evaluated the relationship of our studied scoring systems with patients' triage level, disposition and hospital length of stay. Patients with higher scores in both HEART

Table 2. Relationship of study variables with MACE score

\begin{tabular}{|c|c|c|c|c|}
\hline Variable & & MACE not happened & MACE happened & $P$ value \\
\hline \multirow{3}{*}{ Triage level, No. (\%) } & 1 & $2(40.0)$ & $3(60.0)$ & \multirow{3}{*}{0.019} \\
\hline & 2 & $137(74.9)$ & $46(25.1)$ & \\
\hline & 3 & $12(100)$ & $0(0.0)$ & \\
\hline Hospital length of stay (day), Mean \pm SD & & $2.8 \pm 2.6$ & $6.2 \pm 5.4$ & $<0.001$ \\
\hline \multirow{3}{*}{ HEART score, No. $(\%)$} & $\leq 3$ & $87(97.8)$ & $2(2.2)$ & \multirow{3}{*}{$<0.001$} \\
\hline & $4-6$ & $58(63.0)$ & $34(37.0)$ & \\
\hline & $\geq 7$ & $6(31.6)$ & $13(68.4)$ & \\
\hline HEART score, Mean \pm SD & & $3.35 \pm 1.67$ & $5.65 \pm 1.30$ & $<0.001$ \\
\hline \multirow{3}{*}{ TIMI score, No. (\%) } & $0-2$ & 95 (89.6) & $11(10.4)$ & \multirow{3}{*}{$<0.001$} \\
\hline & $3-4$ & $37(61.7)$ & $23(38.3)$ & \\
\hline & $5-7$ & $1(10.0)$ & $9(90.0)$ & \\
\hline $\mathrm{TIMI}$ score, Mean $\pm \mathrm{SD}$ & & $1.55 \pm 1.36$ & $3.33 \pm 1.32$ & $<0.001$ \\
\hline
\end{tabular}


Table 3. Validity of HEART vs TIMI score in MACE prediction

\begin{tabular}{|c|c|c|c|c|c|}
\hline \multirow{2}{*}{$\begin{array}{l}\text { Validity } \\
95 \% \mathrm{CI}\end{array}$} & \multicolumn{3}{|c|}{ Cut-off points of HEART score } & \multicolumn{2}{|c|}{ Cut-off points of TIMI score } \\
\hline & $\geq 4$ & $\geq 5$ & $\geq 7$ & $\geq 3$ & $\geq 5$ \\
\hline Sensitivity & $95.92(86.099 .5)$ & $81.63(68.091 .2)$ & $26.53(14.941 .1)$ & $74.42(58.8$ 86.5) & $20.93(10.036 .0)$ \\
\hline Specificity & $57.62(49.365 .6)$ & $72.85(65.079 .8)$ & 96.03 (91.6 98.5) & $71.43(63.078 .9)$ & $99.25(95.9100 .0)$ \\
\hline Positive predictive value & $42.3(33.052 .1)$ & $49.4(38.160 .7)$ & $68.4(43.487 .4)$ & 45.7 (33.7 58.1) & $90.0(55.599 .7)$ \\
\hline Negative predictive value & $97.8(92.199 .7)$ & $92.4(86.196 .5)$ & 80.1 (73.5 85.7) & $89.6(82.294 .7)$ & $79.5(72.685 .4)$ \\
\hline
\end{tabular}

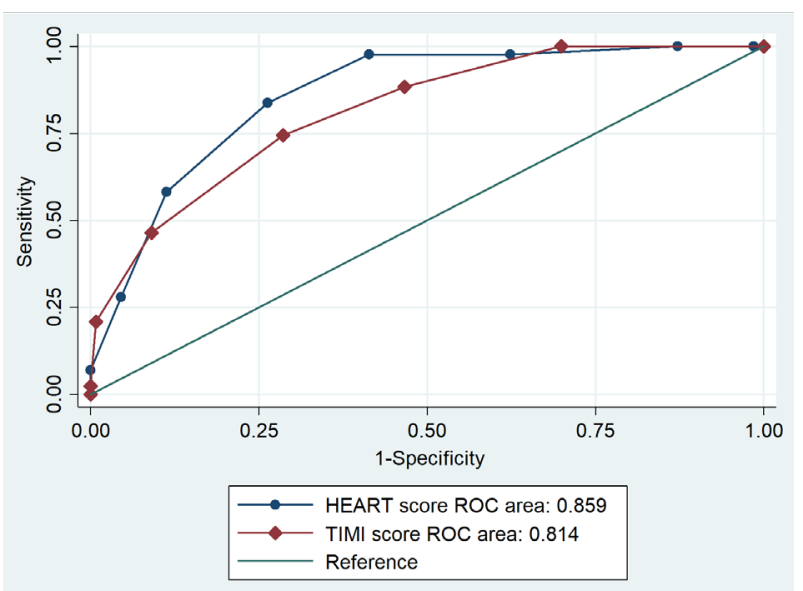

Figure 2. Area under ROC curve of HEART vs TIMI score in MACE prediction.

and TIMI were all admitted and managed in either triage level 1 or $2(P<0.001)$. Patients with higher scores in both HEART and TIMI were significantly more admitted in either cardiac ward or CCU $(P<0.001)$ (Table 4).

There was a statistically significant correlation between scoring systems and hospital length of stay $(P<0.05)$. Pearson correlation coefficients were 0.439 for HEART score and 0.396 for TIMI score.

\section{Discussion}

In the present study, we enrolled 200 suspected cases of ACS during 6 months. It was revealed that the mean \pm SD hospital length of stay was $3.6 \pm 2.0$ days and ACS patients with positive MACE were hospitalized longer than patients with negative MACE. Most of our ACS patients as well as most of cases with positive MACE were males. This result is consistent with the findings of other studies $(15,18)$.

Most of our patients (91.5\%) were admitted at triage level 2. There was a significant difference between MACE score and triage level. The prevalence of MACE at triage level 1 was $60 \%$ and MACE occurrence was less common in lower acuity triage level (level 2 and 3).

We observed that $75.5 \%$ of our cases had negative MACE, while $17 \%$ PCI, $6 \%$ CABG, $1 \%$ cardiac death and 0.5\% AMI happened. Poldervaart et al in 2017 determined that MACE occurred in $19 \%$ of their cases, while $43 \%$ had PCI, $14 \%$ had CABG, $3 \%$ had AMI and only 1 patient died of cardiovascular reason (15).

In our study, it was declared that patients with positive MACE had significantly higher scores in both HEART and TIMI scores. The mean score of HEART in patients with MACE occurrence was 2.4 times more than its mean score in patients with negative MACE. The same result was observed in previous studies. Six et al in 2008, found a HEART score of 6.51 in positive MACE and a score of 3.71 in negative MACE (3). Backus et al in 2013, estimated a HEART score of 6.54 in positive MACE, while the score was 3.96 in negative MACE (19). In Bolvardi et al study, a similar result was observed: 7.42 in positive MACE and 5.42 in negative MACE (2).

Analytical results of our study showed that the mean TIMI score in patients with the endpoint of MACE happening was 1.8 times more than the score in negative MACE. TIMI score in the study conducted by Backus et al was estimated to be 3.68 in positive MACE and 2.21 in negative MACE (19). Sun et al calculated TIMI scores of

Table 4. Mean \pm SD of HEART and TIMI score by triage level and disposition type

\begin{tabular}{|c|c|c|c|c|c|}
\hline Variable & & HEART & P-value & TIMI & $P$ value \\
\hline \multirow{3}{*}{ Triage level } & 1 & $5.40 \pm 1.52$ & \multirow{3}{*}{$<0.001$} & $1.67 \pm 0.58$ & \multirow{3}{*}{$<0.001$} \\
\hline & 2 & $4.04 \pm 1.79$ & & $2.11 \pm 1.53$ & \\
\hline & 3 & $1.33 \pm 0.89$ & & $0.18 \pm 0.40$ & \\
\hline \multirow{4}{*}{ Disposition } & Discharge in less than 24 hours & $2.66 \pm 1.42$ & \multirow{4}{*}{$<0.001$} & $1.12 \pm 1.17$ & \multirow{4}{*}{$<0.001$} \\
\hline & Admission at emergency department & $3.70 \pm 1.25$ & & $1.43 \pm 1.27$ & \\
\hline & Admission at cardiac ward & $4.45 \pm 1.57$ & & $2.34 \pm 1.45$ & \\
\hline & Admission at cardiac care unit & $5.85 \pm 1.87$ & & $3.29 \pm 1.36$ & \\
\hline
\end{tabular}


2.11 in MACE positive group and 1.28 in MACE negative group (20).

Our findings proved that cases with HEART score of 3 or less might have almost $98 \%$ chance of not having MACE and there might be less probability of further workup. Six et al. in 2008 evaluated 122 patients and they showed that in patients with HEART score 0-3 there would be a risk of $2.5 \%$ for MACE occurrence. When HEART score was 4-6 this rate was $20.3 \%$ and in HEART score $\geq 7$ MACE had a chance of $72.7 \%$ (3). Balvardi et al in 2016 evaluated 100 cases and their results revealed that in HEART score $0-3$, MACE occurred in 0\% of patients, in HEART scores 4- 6 and $\geq 7$ MACE happened in $14.5 \%$ and $46.4 \%$ of cases, respectively (2). Jain et al. in 2016 announced their results as follows: $0.6 \%$ in HEART score $0-3,9.5 \%$ in $4-6$ and $38 \%$ in $\geq 7$ (21). In other studies by Backus et al in 2011 and 2016, we observed the same results and they found a chance of more than $60 \%$ for MACE in HEART score $\geq 7$ $(5,22)$.

We found out that HEART score $\geq 4$ was the cut-off point in MACE prediction with the highest sensitivity and HEART score $\geq 7$ was the cut-off point in MACE prediction with the highest specificity. Sun et al showed that HEART score with the cut-off point $>3$ had a sensitivity of $85.8 \%$, and a specificity of $51.2 \%$ (20). Visser et al calculated a sensitivity of $93 \%$ and a specificity of $44 \%$ for $0-3$ HEART score cut-off point. They also reported a sensitivity of $52 \%$ and a specificity of $90 \%$ for 7-10 HEART score cut-off point (23). The same results with high sensitivity for lower HEART score and high specificity for higher HEART score were determined by other studies $(18,24)$.

We also detected that patients with lower TIMI score had less chance of MACE occurrence. Even in TIMI score of 0-2 (low risk patients), there was a $10.4 \%$ probability of MACE. This rate was more than the rate in low risk HEART score. Our finding supported this idea that HEART score might have a better predictive value in low risk patients in comparison to TIMI score when we are assessing MACE.

Sun et al concluded that cases with TIMI score of 0-2 had a $14.4 \%$ chance of MACE (20).

In the present study, it was perceived that TIMI score $\geq 3$ was the best cut-off point in MACE prediction with the highest sensitivity and TIMI score $\geq 5$ was the cut-off point in MACE prediction with the highest specificity. Six et al stated that TIMI score $\geq 1$ had a sensitivity of $87.4 \%$ and a specificity of $47.5 \%$ in MACE prediction (18). Manini et al in 2007, remarked on a sensitivity of $35 \%$ and a specificity of $85 \%$ in ACS prediction (25). The research by Poldervaart et al reported a sensitivity of $95 \%$ and a negative predictive value of $97 \%$ for TIMI score $=0$ (15). The overall findings showed that validity indicators of TIMI score in low risk patients (in lower cut-off points) for MACE prediction were not significant because they had a low sensitivity.

Comparing the predictive value of HEART score versus TIMI score for MACE manifested that the former score had AUC of 0.850, while the latter had AUC of 0.814 . HEART score was a better predictive value but this difference was not significant. Poldevaart et al estimated an AUC of 0.86 for HEART score and an AUC of 0.80 for TIMI score (15). In the prospective study by Chen et al. in 2016, it was demonstrated that HEART score had AUC of 0.726 and TIMI score had AUC of 0.700 . This supported our findings that HEART score had a more powerful predictive value (24). C-statistic predicted probability of HEART score was roughly approximated to be 0.9 for HEART score and 0.6 for TIMI score (22).

We figured the best cut-off point of MACE prediction for HEART and TIMI scores to be $\geq 5$ and $\geq 3$, respectively. Chen et al. enumerated a sensitivity of $48.9 \%$ and a specificity of $83.7 \%$ for HEART score in 30 -day MACE prediction at the optimal cut-off value $>5$ (24).

In this research, we also added that patients with higher scores in both HEART and TIMI were all admitted and managed in either triage level 1 or 2. Patients with higher scores in both HEART and TIMI were significantly more admitted in either cardiac ward or CCU. There was a statistically significant correlation between scoring systems and hospital length of stay. By using a simpler, faster yet more sensitive scoring system like HEART score in an ED, we can predict the outcome of ACS patients and disposition much better.

\section{Limitations of the study}

One limitation we faced in our study was that medical documentations were not accurate and complete in some cases. In addition, the follow up of patients was difficult after 1 month of admission.

\section{Conclusion}

The mean of HEART and TIMI scores in MACE positive cases were significantly more than MACE negative cases. HEART score had a higher sensitivity in low risk patients in MACE prediction in comparison to TIMI score. The best cut-off point was estimated to be $\geq 5$. By increasing the acuity of patient's condition (level 1 and 2 triage), we should expect higher HEART score and worse MACE outcome. Patients with higher HEART score were admitted longer at the hospital and there was a more probability of being admitted at CCU.

\section{Authors' contributions}

$\mathrm{AA}$ and EV conceived the study, designed the trial, supervised the conduct of the trial and collected data. MB and EV undertook the recruitment of participating centers 
and patients and managed the data, including quality control. AA and EV provided statistical advice on study design and analyzed the data. EV drafted the manuscript, $\mathrm{AJ}$ and JS also undertook recruitment of participating centers and patients and managed the data, including quality control. and all authors contributed substantially to its revision. EV takes responsibility for the paper as a whole.

\section{Ethical issues}

The present study was approved by the ethics committee of TUMS (ID: IR.TUMS.IKHC.REC.1396.3888). All patients enrolled in this study were given a brief report of the study process and informed written consent was granted.

\section{References}

1. Niska R, Bhuiya F, Xu J. National Hospital Ambulatory Medical Care Survey: 2007 emergency department summary. Natl Health Stat Report 2010(26):1-31.

2. Bolvardi E, Raoufi P, Vakili V, Jahed Taherani H, Movaffaghi M, Bahramian M, et al. Evaluation efficacy of HEART score in prediction of major advanced cardiac events in patients with chest pain. Biosci Biotechnol Res Asia 2016;13(2):9991005. doi:10.13005/bbra/2126

3. Six AJ, Backus BE, Kelder JC. Chest pain in the emergency room: value of the HEART score. Neth Heart J 2008;16(6):191-6. doi:10.1007/bf03086144

4. Amsterdam EA, Kirk JD, Bluemke DA, Diercks D, Farkouh ME, Garvey JL, et al. Testing of low-risk patients presenting to the emergency department with chest pain: a scientific statement from the American Heart Association. Circulation 2010;122(17):1756-76. doi:10.1161/CIR.0b013e3181ec61df

5. Six AJ, Backus BE, Kingma A, Kaandorp SI. Consumption of diagnostic procedures and other cardiology care in chest pain patients after presentation at the emergency department. Neth Heart J 2012;20(12):499-504. doi:10.1007/s12471-012-0322-6

6. Hoffmann U, Truong QA, Schoenfeld DA, Chou ET, Woodard PK, Nagurney JT, et al. Coronary CT angiography versus standard evaluation in acute chest pain. N Engl J Med 2012;367(4):299-308. doi:10.1056/NEJMoa1201161

7. National Clinical Guideline Centre for Acute and Chronic Conditions. Chest Pain of Recent Onset: Assessment and Diagnosis of Recent Onset Chest Pain or Discomfort of Suspected Cardiac Origin. London: National Clinical Guideline Centre for Acute and Chronic Conditions; 2010.

8. Eslick GD. Classification, natural history, epidemiology, and risk factors of noncardiac chest pain. Dis Mon 2008;54(9):593-603. doi:10.1016/j.disamonth.2008.06.003

9. Chan GW, Sites FD, Shofer FS, Hollander JE. Impact of stress testing on 30-day cardiovascular outcomes for lowrisk patients with chest pain admitted to floor telemetry beds. Am J Emerg Med 2003;21(4):282-87. doi:10.1016/ s0735-6757(03)00080-9

10. Davis B. Rosen's emergency medicine: Concepts and clinical practice. Prehospital Emergency Care 2004;8(3):334-5.

11. Antman EM, Cohen M, Bernink PJ, McCabe CH, Horacek
T, Papuchis G, et al. The TIMI risk score for unstable angina/non-ST elevation MI: a method for prognostication and therapeutic decision making. JAMA 2000;284(7):83542. doi:10.1001/jama.284.7.835

12. Fox KA, Eagle KA, Gore JM, Steg PG, Anderson FA. The global registry of acute coronary events, 1999 to 2009--GRACE. Heart 2010;96(14):1095-101. doi:10.1136/ hrt.2009.190827

13. Hermans WR, Foley DP, Rensing BJ, Rutsch W, Heyndrickx GR, Danchin N, et al. Usefulness of quantitative and qualitative angiographic lesion morphology, and clinical characteristics in predicting major adverse cardiac events during and after native coronary balloon angioplasty. CARPORT and MERCATOR Study Groups. Am J Cardiol 1993;72(1):14-20. doi:10.1016/0002-9149(93)90211-t

14. Keane D, Buis B, Reifart N, Plokker TH, Ernst JM, Mast EG, et al. Clinical and angiographic outcome following implantation of the new less shortening wallstent in aortocoronary vein grafts: introduction of a second generation stent in the clinical arena. J Interv Cardiol 1994;7(6):557-64. doi:10.1111/j.1540-8183.1994.tb00496.x

15. Poldervaart JM, Langedijk M, Backus BE, Dekker IMC, Six AJ, Doevendans PA, et al. Comparison of the GRACE, HEART and TIMI score to predict major adverse cardiac events in chest pain patients at the emergency department. Int J Cardiol 2017;227:656-61. doi:10.1016/j. ijcard.2016.10.080

16. World Health Organization (WHO). The World Health Report 2002: Reducing Risks, Promoting Healthy Life. WHO; 2002.

17. Faxon DP, Creager MA, Smith SC Jr, Pasternak RC, Olin JW, Bettmann MA, et al. Atherosclerotic Vascular Disease Conference: executive summary: atherosclerotic Vascular Disease Conference proceeding for healthcare professionals from a special writing group of the American Heart Association. Circulation 2004;109(21):2595-604. doi:10.1161/01.cir.0000128517.52533.db

18. Six AJ, Cullen L, Backus BE, Greenslade J, Parsonage W, Aldous S, et al. The HEART score for the assessment of patients with chest pain in the emergency department: a multinational validation study. Crit Pathw Cardiol 2013;12(3):121-6. doi:10.1097/HPC.0b013e31828b327e

19. Backus BE, Six AJ, Kelder JC, Bosschaert MA, Mast EG, Mosterd A, et al. A prospective validation of the HEART score for chest pain patients at the emergency department. Int J Cardiol 2013;168(3):2153-8. doi:10.1016/j. ijcard.2013.01.255

20. Sun BC, Laurie A, Fu R, Ferencik M, Shapiro M, Lindsell CJ, et al. Comparison of the HEART and TIMI risk scores for suspected acute coronary syndrome in the emergency department. Crit Pathw Cardiol 2016;15(1):1-5. doi:10.1097/hpc.0000000000000066

21. Jain T, Nowak R, Hudson M, Frisoli T, Jacobsen G, McCord J. Short- and long-term prognostic utility of the HEART score in patients evaluated in the emergency department for possible acute coronary syndrome. Crit Pathw Cardiol 2016;15(2):40-5. doi:10.1097/hpc.0000000000000070

22. Backus BE, Six AJ, Kelder JH, Gibler WB, Moll FL, 
Doevendans PA. Risk scores for patients with chest pain: evaluation in the emergency department. Curr Cardiol Rev 2011;7(1):2-8. doi:10.2174/157340311795677662

23. Visser A, Wolthuis A, Breedveld R, ter Avest E. HEART score and clinical gestalt have similar diagnostic accuracy for diagnosing ACS in an unselected population of patients with chest pain presenting in the ED. Emerg Med J 2015;32(8):595-600. doi:10.1136/emermed-2014-203798

24. Chen XH, Jiang HL, Li YM, Chan CPY, Mo JR, Tian
CW, et al. Prognostic values of 4 risk scores in Chinese patients with chest pain: prospective 2-centre cohort study. Medicine (Baltimore) 2016;95(52):e4778. doi:10.1097/ md.0000000000004778

25. Manini AF, Dannemann N, Brown DF, Butler J, Bamberg F, Nagurney JT, et al. Limitations of risk score models in patients with acute chest pain. Am J Emerg Med 2009;27(1):43-8. doi:10.1016/j.ajem.2008.01.022 